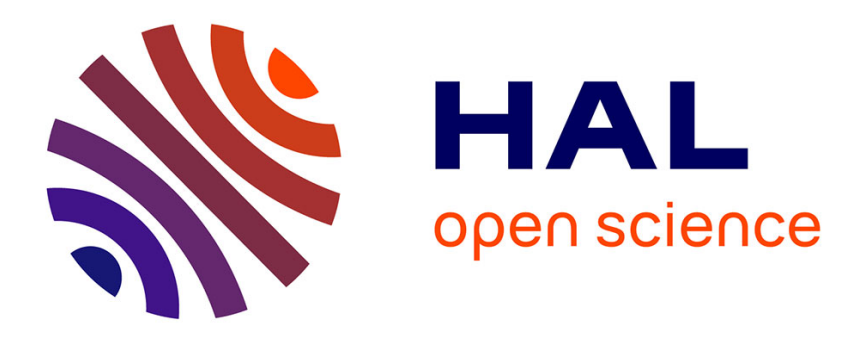

\title{
Quadratic hedging in an incomplete market derived by an influent informed investor
}

\author{
Anne Eyraud-Loisel
}

\section{To cite this version:}

Anne Eyraud-Loisel. Quadratic hedging in an incomplete market derived by an influent informed investor. Stochastics: An International Journal of Probability and Stochastic Processes, 2013, 10.1080/17442508.2011.652632 . hal-00450949

\section{HAL Id: hal-00450949 \\ https://hal.science/hal-00450949}

Submitted on 27 Jan 2010

HAL is a multi-disciplinary open access archive for the deposit and dissemination of scientific research documents, whether they are published or not. The documents may come from teaching and research institutions in France or abroad, or from public or private research centers.
L'archive ouverte pluridisciplinaire HAL, est destinée au dépôt et à la diffusion de documents scientifiques de niveau recherche, publiés ou non, émanant des établissements d'enseignement et de recherche français ou étrangers, des laboratoires publics ou privés. 


\title{
Quadratic hedging in an incomplete market derived by an influent informed investor
}

\author{
Anne EYRAUD-LOISEL
}

27 January 2010

\begin{abstract}
In this paper a model with an influent and informed investor is presented. The studied problem is the point of view of a non informed agent hedging an option in this influenced and informed market. Her lack of information makes the market incomplete to the non informed agent. The obtained results, by means of Malliavin calculus and Clark-Ocone Formula, as well as Filtering Theory are the expressions and a comparison between the strategy of the non informed trader, and the strategy of the informed agent. An expression of the residual risk a non informed trader keeps by detaining an option in this influenced and informed market is derived using a quadratic approach of hedging in incomplete market. Finally, the analysis leads to a measure of the lack of information that makes the incompleteness of the market. The financial interpretation is explained throughout the theoretical analysis, together with an example of such influenced informed model.
\end{abstract}

Keywords Enlargement of filtration · FBSDE · quadratic hedging · risk minimization · insider trading · influent investor · asymmetric information · martingale representation - Clark-Ocone formula.

AMS Classification (2000): 60H10, 60G44, 60H07, 60J75, 91G20, 91B70, 93E11.

JEL Classification: C60,G11,G14.

\footnotetext{
A. Eyraud-Loisel

Université de Lyon, Université Lyon 1, ISFA, Laboratoire SAF

50 avenue Tony Garnier 69007 LYON

Tel +33-4-37-28-74-35 E-mail: anne.eyraud@univ-lyon1.fr
} 


\section{Introduction}

This article deals with a hedging problem in an incomplete market, where the incompleteness of the market is due to the presence of an influent informed investor. This influent informed agent is supposed to possess an additional information, and is also supposed to influence the market. This is a natural extension of the work of Eyraud-Loisel [12], where the informed agent is supposed to be a small investor, who does not influence asset prices. EyraudLoisel (2009) [13] studies the case of an influent informed agent, who wants to hedge an option.

The chosen model is the same as in [13], where the market prices process is influenced by the portfolio of an informed agent. It is more realistic to consider large traders in the market, who may influence the evolution of asset prices, either by their large investment depth, or by their notoriety, when a charter phenomenon appears. Then asset prices may be influenced by certain big agents in the market, and it is quite natural to suppose that such large agents may have more easily access to additional information on the market. This is the reason why it is interesting to develop a model with an influent informed investor. The additional information is supposed to be a strong initial information, modeled by an initial enlargement of the Brownian filtration, as developed for insider trader modeling in Grorud and Pontier [19], or Amendinger [1]. This influenced market has been already studied in [13] from the influent informed agent's point of view, by solving the FBSDE appearing when modeling the hedging problem of the large trader, under the enlarged filtration induced by the additional information. The present article is devoted to the study of the non-informed agent's strategy in this influenced market. Despite the completeness of the influenced informed market, the non-informed market is incomplete : the common agent does not have access to the entire information driving the prices process.

In Section 2, we present the model and quickly recall the results of [13] about the hedging strategy of the influent informed agent, and the completeness of the influenced informed market.

In Section 3 we study the incompleteness of the market from a non informed trader's point of view. As a consequence of the influence of the large informed trader, the filtration generated by prices reflect a part of the additional information, and is not the Brownian filtration anymore. In order to compare both strategies, of the informed influent agent, and of the small common agent, we use a quadratic hedging approach in this incomplete market. As there is no predictable representation property in this market, we use the local risk minimization approach, introduced by Föllmer and Sonderman [16] in the case where prices are martingales, and developed by Schweizer [33] when prices are only semi-martingales, and Föllmer and Schweizer [15] in terms of minimal martingale measure. This approach shows the existence of a unique risk-minimizing strategy, which may be expressed using the Galtchouk-KunitaWatanabe decomposition (as developed in Ansel and Stricker [2]). Another possible approach would be the mean-variance hedging method, developed by 
Gouriéroux, Laurent and Pham [18], and Rheinlander and Schweizer [32], who obtained an expression of the optimal strategy in terms of variance-optimal martingale measure. A very clear review of the subject and of these two quadratic hedging methods in a continuous market is provided in Pham (2000) [30].

We have chosen the local risk-minimization approach developed in Föllmer and Schweizer (1991) [15], as our model fits into their global idea : considering an incomplete market where the incompleteness is due to a lack of information. In our model, when adding the additional information, the market is complete. We compare the hedging strategy of a non informed agent to the strategy of the informed influent agent, in an incomplete market, under Hypothesis $\left(\mathbf{H}_{3}\right)$, which differs from classical hedging hypotheses studied in Föllmer and Schweizer [15]. We obtain an expression of the optimal strategy in terms of projection of the strategy of the informed influent agent, with respect of the filtration generated by prices (Filtering Theory is used). Moreover, we give a version of the Clark-Ocone formula in our framework of enlarged filtration, as well as an expression of the informed agent strategy in terms of Malliavin derivatives. We derive also an expression of the residual risk linked to the lack of information of the non informed agent. In the last section, we give an example of information and influence that satisfies all hypotheses of this model, and give the expressions of the strategies and residual risk of the lack of information of the non informed trader derived in the previous section and applied in this case.

\section{Model}

We recall here the model introduced in Eyraud-Loisel [13], where an informed and influent agent wants to hedge against a given contingent claim. The market is supposed to be driven by a multidimensional Brownian motion. The wealth process $X_{t}$ and investment strategy $\pi_{t}$ of the influent agent are expressed as the solution of a backward stochastic differential equation. This investor may influence asset prices either by his wealth $X_{t}$, or by his portfolio strategy $\pi_{t}$, which may influence the drift $b$ of the volatility $\sigma$ of prices. The BSDE driving the wealth process and the investment strategy, modeling the hedging problem, is then fully coupled with the forward equation of prices. The equation to be solved for the hedging problem is the following forwardbackward stochastic differential equation (FBSDE):

$$
\left\{\begin{array}{l}
P_{t}=P_{0}+\int_{0}^{t} b\left(s, P_{s}, X_{s}, Z_{s}\right) d s+\int_{0}^{t}<\sigma\left(s, P_{s}, X_{s}, Z_{s}\right), d W_{s}> \\
X_{t}=X_{T}-\int_{t}^{T} f\left(s, P_{s}, X_{s}, Z_{s}\right) d s-\int_{t}^{T}<Z_{s}, d W_{s}>
\end{array}\right.
$$

where $b\left(s, P_{s}, X_{s}, Z_{s}\right)=P_{s} b\left(s, P_{s}, X_{s}, Z_{s}\right), \sigma\left(s, P_{s}, X_{s}, Z_{s}\right)=P_{s} \sigma^{\prime}\left(s, P_{s}, X_{s}, Z_{s}\right)$, and $Z=\sigma^{* *}\left(s, X_{s}, P_{s}, \pi_{s}\right) \pi_{s}$.

As the influent agent has an additional information on the market, this FBSDE has to be solved under an initial enlargement of filtration $\mathcal{Y}_{t}$ of the Brownian 
filtration $\mathcal{F}_{t}$. The additional information is modeled by a random variable $L \in \mathcal{F}_{T^{\prime}}$, where $T^{\prime}>T$, and the enlarged filtration is $\mathcal{Y}_{t}:=\bigcap_{s>t}\left(\mathcal{F}_{s} \vee \sigma(L)\right)$. The information is supposed to satisfy usual hypothesis $\left(H_{3}\right)$, first introduced by Jeulin [25,26], and extensively used by Grorud and Pontier [19], Amendinger [1] and Eyraud-Loisel [12] :

Definition 2.1 Hypothesis $\left(\mathbf{H}_{3}\right)$

There exists a probability measure $\mathbb{Q}$ equivalent to $\mathbb{P}$ such that under $\mathbb{Q}, \mathcal{F}_{t}$ and $\sigma(L)$ independent for all $t<T^{\prime}$.

The following assumptions on the coefficients (standard Lipschitz, linear growth and integrability conditions) are made:

(A1) $\sigma$ is invertible $d t \times d \mathbb{P}$-a.s., $\sigma^{\prime}$ and $\sigma^{\prime-1}\left(b^{\prime}-r\right)$ are bounded.

(A2) Functions $b, f, \sigma, g$ are continuous w.r.t. $p, x, z$ in $\mathbb{R}^{k} \times \mathbb{R} \times \mathbb{R}^{k}$, for all $(\omega, t) \in \Omega \times[0, T]$

(A3) $\exists \lambda_{1}, \lambda_{2} \in \mathbb{R}$ such that $\forall t, p, p_{1}, p_{2}, x, x_{1}, x_{2}, z, \mathbb{P}$-a.s. :

$$
\begin{gathered}
<b\left(t, p_{1}, x, z\right)-b\left(t, p_{2}, x, z\right), p_{1}-p_{2}>\leq \lambda_{1}\left|p_{1}-p_{2}\right|^{2}, \\
<f\left(t, p, x_{1}, z\right)-f\left(t, p, x_{2}, z\right), x_{1}-x_{2}>\leq \lambda_{2}\left|x_{1}-x_{2}\right|^{2} .
\end{gathered}
$$

(A4) $b$ is globally Lipschitz w.r.t. $x$ and $z$, and at most linearly increasing w.r.t. $p$, and $f$ is globally Lipschitz w.r.t. $p$ and $z$, and at most linearly increasing w.r.t. $x: \exists k, k_{i}$ such that $\mathbb{P}$-a.s. $\forall t, p_{i}, x_{i}, z_{i}$

$$
\begin{aligned}
\left|b\left(t, p, x_{1}, z_{1}\right)-b\left(t, p, x_{2}, z_{2}\right)\right| & \leq k_{1}\left|x_{1}-x_{2}\right|+k_{2}\left\|z_{1}-z_{2}\right\|, \\
|b(t, p, x, z)| & \leq|b(t, 0, x, z)|+k(1+|p|), \\
\left|f\left(t, p_{1}, x, z_{1}\right)-f\left(t, p_{2}, x, z_{2}\right)\right| & \leq k_{3}\left|p_{1}-p_{2}\right|+k_{4}\left\|z_{1}-z_{2}\right\|, \\
|f(t, p, x, z)| & \leq|f(t, p, 0, z)|+k(1+|x|) .
\end{aligned}
$$

(A5) $\sigma$ is globally Lipschitz w.r.t. $p, x$ and $z: \exists k_{i}$ such that $\mathbb{P}$-a.s. $\forall t, p_{i}, x_{i}, z_{i}$

$$
\left\|\sigma\left(t, p_{1}, x_{1}, z_{1}\right)-\sigma\left(t, p_{2}, x_{2}, z_{2}\right)\right\|^{2} \leq k_{5}^{2}\left|p_{1}-p_{2}\right|^{2}+k_{6}^{2}\left|x_{1}-x_{2}\right|^{2}+k_{7}^{2}\left\|z_{1}-z_{2}\right\|^{2} .
$$

(A6) $g$ is globally Lipschitz w.r.t. $p: \exists k_{8}$ such that $\mathbb{P}$-a.s. $\forall p_{i}$

$$
\left|g\left(p_{1}\right)-g\left(p_{2}\right)\right|^{2} \leq k_{8}^{2}\left|p_{1}-p_{2}\right|^{2} .
$$

(A7) $\forall p, x, z, b(., p, x, z), f(., p, x, z)$ and $\sigma(., p, x, z)$ are $\mathcal{F}$-adapted processes and $g(p)$ is $\mathcal{F}_{T}$-measurable. Moreover :

$$
E_{\mathbb{P}} \int_{0}^{T}|b(s, 0,0,0)|^{2} d s+E_{\mathbb{P}} \int_{0}^{T}|f(s, 0,0,0)|^{2} d s+E_{\mathbb{P}} \int_{0}^{T}\|\sigma(s, 0,0,0)\|^{2} d s+E_{\mathbb{P}}|g(0)|^{2}<+\infty .
$$

(A7') Suppose also

$$
E_{\mathbb{Q}} \int_{0}^{T}|b(s, 0,0,0)|^{2} d s+E_{\mathbb{Q}} \int_{0}^{T}|f(s, 0,0,0)|^{2} d s+E_{\mathbb{Q}} \int_{0}^{T}\|\sigma(s, 0,0,0)\|^{2} d s+E_{\mathbb{Q}}|g(0)|^{2}<+\infty .
$$


(A8) $b$ is also supposed to be globally Lipschitz w.r.t. $p: \exists k_{9}$ such that $\mathbb{P}$-a.s. $\forall t, p_{i}, x, z$

$$
\left|b\left(t, p_{1}, x, z\right)-b\left(t, p_{2}, x, z\right)\right|^{2} \leq k_{9}^{2}\left|p_{1}-p_{2}\right|^{2} .
$$

We suppose also that one of the 3 following cases (I1) to (I3) is satisfied :

(I1) The influence is weak : the forward and backward equations are weakly coupled: $\exists \varepsilon_{0}>0$ depending on $k_{3}, k_{4}, k_{5}, k_{8}, \lambda_{1}, \lambda_{2}$ and $T$ such that $k_{1}, k_{2}, k_{6}, k_{7} \in\left[0, \varepsilon_{0}\right)$.

(I2) $g, \mathcal{F}_{T}$-measurable, is independent from the price process, and $\lambda_{1}$ and $\lambda_{2}$ from hypothesis (A3) satisfy also: $\exists C_{i}>0, i=1,2,3,4, C_{4}<k_{4}^{-1}, \theta>0$ such that

$$
\lambda_{1}+\lambda_{2}<-\frac{1}{2}\left[k_{3} C_{3}\left(\frac{k_{2} C_{2}+k_{7}^{2}}{1-k_{4} C_{4}}+\frac{k_{1} C_{1}+k_{6}^{2}}{\theta}\right)+\frac{k_{1}}{C_{1}}+\frac{k_{2}}{C_{2}}+\frac{k_{3}}{C_{3}}+\frac{k_{4}}{C_{4}}+k_{5}^{2}+\theta\right] .
$$

(I3) $\sigma$ is independent from $z$ : the portfolio does not influence the volatility of prices, and $\lambda_{1}$ and $\lambda_{2}$ from hypothesis (A3) satisfy also: $\exists C_{i}>0, i=$ $1,3,4, C_{4}<k_{4}^{-1}, \theta>0, \alpha>0$ such that

$$
\lambda_{1}+\lambda_{2}<-\frac{1}{2}\left[(1+\alpha)\left[k_{1} C_{1}+k_{6}^{2}+\frac{k_{2}^{2}}{\alpha\left(1-k_{4} C_{4}\right)}\right]\left(k_{8}^{2}+\frac{k_{3} C_{3}}{\theta}\right) \frac{k_{1}}{C_{1}}+\frac{k_{3}}{C_{3}}+\frac{k_{4}}{C_{4}}+k_{5}^{2}+\theta\right] .
$$

Under these hypotheses, Eyraud-Loisel $[14,13]$ derived an existence and uniqueness theorem of such FBSDE under an initial enlargement of filtration satisfying Hypothesis $\left(\mathbf{H}_{3}\right)$ :

Theorem 2.2 (Theorem 1 of [13])

Under Hypotheses $\left(\mathbf{H}_{\mathbf{3}}\right),(A 1)$ to $(A 8)$, and under one of the cases of influence (I1) to (I3), the following forward-backward stochastic differential equation:

$$
\left\{\begin{array}{l}
P_{t}=P_{0}+\int_{0}^{t} b\left(s, P_{s}, X_{s}, Z_{s}\right) d s+\int_{0}^{t}<\sigma\left(s, P_{s}, X_{s}, Z_{s}\right), d W_{s}> \\
X_{t}=\xi-\int_{t}^{T} f\left(s, P_{s}, X_{s}, Z_{s}\right) d s-\int_{t}^{T}<Z_{s}, d W_{s}>
\end{array}\right.
$$

admits in the space $(\Omega, \mathcal{Y}, Q)$ a unique $\mathcal{Y}$-adapted solution $(P, X, Z)$, such that

$$
E_{\mathbb{Q}} \int_{0}^{T}\left(\sup _{0 \leq u \leq t}\left|P_{t}\right|^{2}+\sup _{0 \leq u \leq t}\left|X_{t}\right|^{2}+\left\|Z_{t}\right\|^{2}\right) d t<+\infty .
$$

The FBSDE has a unique adapted solution in the enlarged space. This means that the influent agent has a unique hedging strategy adapted to his information. As volatility is supposed to be invertible, it is possible to derive from $Z_{t}$ the unique portfolio $\pi_{t}$ hedging the pay-off $\xi$. If the solution of the insider trader is adapted to the Brownian filtration, then it is the same as if there was no additional information.

Any contingent claim in $L^{2}(\Omega, \mathcal{Y}, \mathbb{Q})$ is attainable. The market is complete for the informed investor, w.r.t. to the enlarged filtration. The hedging problem in the market is reduced to a resolution of a FBSDE, whose coupling depends on the influence of the insider in the market. The results from the insider's 
point of view are the same as in [12], with no influence.

What is different in the model with an influent agent, is the behavior of the market from the point of view of a normally informed agent (for whom the additional information is unknown). The market is incomplete for the non informed trader. This incompleteness is due to his lack of information, as in Föllmer and Schweizer (1991) [15]. The present model is an example of complete market which becomes incomplete from a small non informed investor point of view. The study of such a market uses tools of quadratic hedging in incomplete market, or under incomplete information, and it is the aim of this paper.

\section{Quadratic hedging in this incomplete market}

\subsection{Introduction of a non informed agent}

In this model, the market prices are influenced by the insider's investment process. We suppose from now on that hypotheses of Theorem 2.2 are satisfied. We consider a non informed agent, who wants to hedge against an option in this market. This non informed agent is supposed to be a small investor, which means that she can not influence asset prices. This can be viewed as a problem of hedging in partial information, or lack of information : the non informed agent can only observe prices. The historic of prices of the risky assets is the only available information. The non informed agent knows the filtration generated by prices :

$$
\tilde{\mathcal{F}}_{t}:=\mathcal{F}_{t}^{P}:=\sigma\left(P_{s}, 0 \leq s \leq t\right)
$$

As the coefficients in the diffusion of prices are adapted to the enlarged filtration, due to the influence of the informed agent, even the Brownian filtration is not available to the small trader. Other processes that appear in the BSDE of the hedging problem of the non informed trader, in particular Brownian motion, are not directly observable on the market. Her portfolio strategy and wealth processes have to be $\tilde{\mathcal{F}}$-adapted. As the investment strategies of the informed and non informed traders are adapted to different filtrations, their sets of admissible strategies differ. Even if the insider market is complete, it may not be complete from the non informed trader's point of view. If the non informed trader has a hedging strategy, it may be different from the strategy of the influent informed trader, whose exact replicating strategy is $\mathcal{Y}$-adapted. Completeness of a market (defined as "any square integrable contingent claim is attainable") depends on the considered filtration and probability measure : everything depends on the existence $\mathrm{f}$ a martingale representation Theorem. In our context, under Hypothesis $\left(H_{3}\right)$, there exists a martingale representation Theorem in the enlarged space $\mathcal{Y}$, under probability $\mathbb{Q}$, and in the Brownian filtration $\mathcal{F}$ under probability $\mathbb{P}$, but not under filtration $\tilde{\mathcal{F}}$ (see Jacod and Shiryaev [23] Eyraud-Loisel [12]). The problem becomes a hedging problem 
in an incomplete market. We will use a quadratic local risk-minimization approach for this study.

We first study in Subsection 3.2 Proposition 3.5 the Kunita-Watanabe decomposition of the contingent claim in the prices filtration. In Subsection 3.3 a Clark-Ocone formula is obtained in the enlarged space, from which we derive an expression of the integrand of the representation Theorem in this space. Finally we give an expression of the residual quadratic risk linked to the lack of information of the non informed trader, successively under two families of risk-neutral probabilities : the risk-neutral probabilities from the informed trader's point of view in Paragraph 3.4.1, and the risk-neutral probabilities from the non informed trader's point of view in Paragraph 3.4.2.

\subsection{Hedging strategy and Kunita-Watanabe decomposition}

The different considered $\sigma$-fields are linked by the following relations :

$$
\begin{gathered}
\tilde{\mathcal{F}}_{t} \subset \mathcal{Y}_{t}, \text { and } \mathcal{F}_{t} \subset \mathcal{Y}_{t} \\
\mathcal{Y}_{t}=\bigcap_{s>t} \mathcal{F}_{s} \vee \sigma(L)=\bigcap_{s>t} \tilde{\mathcal{F}}_{s} \vee \sigma(L) .
\end{gathered}
$$

To keep notations as light as possible, we consider only one risky asset here, but all results may be generalized to the $d$-dimensional case. The dynamics of the risky asset satisfies the following forward equation :

$$
P_{t}=P_{0}+\int_{0}^{t} P_{s} b^{\prime}\left(s, P_{s}, X_{s}^{L}, \pi_{s}^{L}\right) d s+\int_{0}^{t} P_{s} \sigma^{\prime}\left(s, P_{s}, X_{s}^{L}, \pi_{s}^{L}\right) d W_{s}
$$

where $P_{s}, X_{s}^{L}$ and $\pi_{s}^{L}$ are respectively the price of the risky asset, and the wealth and hedging strategy of the informed agent, deduced from the unique solution of FBSDE (2.1) in the enlarged space (Theorem 2.2).

The non informed trader wants to hedge against a European contingent claim $H=h\left(P_{T}\right) \in \mathcal{L}^{2}(\Omega, \tilde{\mathcal{F}}, \mathbb{Q})$. This contingent claim is not necessarily the same as the contingent claim replicated by the informed trader. This point of view may be used to compare investments strategies of two small agents, one who has access to the additional information and the other who can only observe prices and has just a part of the total information. If the same contingent claim is considered, it may lead to a comparison of the strategies of both agents, the first one being influent and informed and the other one not influent and not informed.

Under $\mathbb{Q}$, probability measure defined in the previous section (Definition 2.1) Hypothesis $\left(\mathbf{H}_{\mathbf{3}}\right)$ is satisfied, $W_{t}$ is a $\mathcal{Y}$-Brownian motion, and $P_{t}$ is a $(\mathcal{Y}, Q)$-semimartingale. As $P_{t}$ is $\tilde{\mathcal{F}}$-adapted, it is also a $(\tilde{\mathcal{F}}, \mathbb{Q})$-semimartingale. The market is not complete: as $H$ is attainable in $(\mathcal{Y}, \mathbb{Q})$, the replicating strategy is adapted to filtration $\mathcal{Y}$, and so is not admissible for the non informed trader in the smaller space. We will develop quadratic hedging arguments in 
order to find the hedging strategy of the non informed trader that minimize the intrinsic residual risk of the contingent claim, risk linked to the lack of information on this influenced informed market.

Denote by $\mathcal{Q}$ the set of all equivalent martingale measures under filtration $\mathcal{Y}$, i.e. the set of all probability measures equivalent to $\mathbb{Q}$ (and so equivalent to $\mathbb{P}$ ) under which $P_{t}$ is a $\mathcal{Y}$-martingale. Financially speaking it is the set of all risk-neutral probability measures from the informed trader's point of view. Denote also by $\mathcal{Q}_{N}$ the set of all equivalent martingale measures under filtration $\tilde{\mathcal{F}}$, equivalent to $\mathbb{P}$, i.e. the set of all probability measures equivalent to

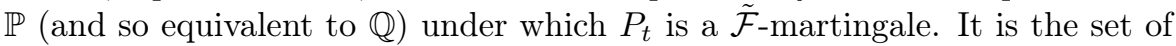
all risk-neutral probability measures from the non informed trader's point of view.

We have clearly

$$
\mathcal{Q} \subset \mathcal{Q}_{N}
$$

Indeed, intuitively, from her lack of information, the non informed agent will have a larger range of risk-neutral probability measures. Some of the riskneutral probability measures will appear to be risk-neutral from the non informed agent's point of view, whereas with the additional information, they will appear not to be risk-neutral. Conversely, any risk-neutral probability measure for the informed trader is always also risk-neutral for the non informed trader: if $P$ is a $\mathcal{Y}$-martingale, as it is $\tilde{\mathcal{F}}$-adapted, it is a $\tilde{\mathcal{F}}$-martingale. Moreover, $\mathcal{Q}$ is not empty: from Assumption $(A 1), \sigma^{\prime}$ is invertible and $\mathcal{E}\left(-\sigma^{\prime-1}\left(b^{\prime}-\right.\right.$ $r) W$ ) is integrable, which are classical arguments implying no arbitrage opportunities (see Delbaen and Schachermayer [11]).

Recall the definition of orthogonality for two martingales (see Protter [31], and Pham [30]):

Definition 3.1 Two martingales $L, N$ are (strongly) orthogonal if their product $L N$ is a local martingale with initial value $L_{0} N_{0}=0$.

If $L$ and $N \in \mathcal{M}_{l o c}^{2}$, this is equivalent to $\langle L, N\rangle=0$.

We now give the definition of the decomposition of Kunita-Watanabe (see Ansel and Stricker [2] or Pham [30]) on a general probability space $(\Omega, \mathcal{F}, \mathbb{P})$ :

Definition 3.2 Let $N$ be a real-valued $(\mathcal{F}, \mathbb{P})$-local martingale, and $M$ a $\mathbb{R}^{d}$-valued $(\mathcal{F}, \mathbb{P})$-local martingale. If $N \in \mathcal{M}_{(\text {loc })}^{2}(\mathcal{F}, \mathbb{P})$ (square-integrable $(\mathcal{F}, \mathbb{P})$-local martingale locale under $\mathbb{P})$ and $M \in \mathcal{M}_{(l o c)}^{2}(\mathcal{F}, \mathbb{P})$, then we have the following projection result, called Kunita-Watanabe decomposition of the $(\mathcal{F}, \mathbb{P})$-martingale $N$ with respect to $M$ :

$$
N_{t}=N_{0}+\int_{0}^{t} \theta_{u} d M_{u}+L_{t}, \quad \mathbb{P}-\text { a.s. } 0 \leq t \leq T
$$

where $\theta \in L_{(l o c)}^{2}(M), L \in \mathcal{M}_{0,(l o c)}^{2}(\mathcal{F}, \mathbb{P})$ (square integrable $(\mathcal{F}, \mathbb{P})$-local martingale with initial value 0$)$ and $L$ orthogonal to $M$ in $(\mathcal{F}, \mathbb{P})$. 
Remark 3.3 Decomposition (3.2) is unique in the sense that if

$$
N_{t}=N_{0}+\int_{0}^{t} \theta_{u} d M_{u}+L_{t}=\tilde{N}_{0}+\int_{0}^{t} \tilde{\theta_{u}} d M_{u}+\tilde{L}_{t}
$$

with $\left(N_{0}, \theta, L\right)$ et $\left(\tilde{N}_{0}, \tilde{\theta}, \tilde{L}\right)$ satisfying the Kunita-Watanabe hypotheses, then $N_{0}=\tilde{N}_{0}, \int_{0}^{t} \theta_{u} d M_{u}=\int_{0}^{t} \tilde{\theta}_{u} d M_{u}$ and $L_{t}=\tilde{L}_{t} \mathbb{P}$-a.s. $\forall 0 \leq t \leq T$.

Remark also that if $M$ and $N$ are in $\mathcal{M}^{2}(\mathcal{F}, \mathbb{P})$, then $\theta \in L^{2}(M)$ and $L \in$ $\mathcal{M}_{0}^{2}(\mathcal{F}, \mathbb{P})$.

Let $\tilde{\mathbb{Q}} \in \mathcal{Q}$ be risk-neutral from the informed agent, $\tilde{\mathbb{Q}}$ equivalent to $\mathbb{Q}$, under which $P$ is a $(\mathcal{Y}, \tilde{\mathbb{Q}})$-martingale. As $P_{t}$ is $\tilde{\mathcal{F}}$-adapted, it is a $(\tilde{\mathcal{F}}, \tilde{\mathbb{Q}})$ martingale.

$H \in \mathcal{L}^{2}(\Omega, \tilde{\mathcal{F}}, \tilde{\mathbb{Q}})$ is attainable with respect to the largest filtration. The martingale representation Theorem in $(\Omega, \mathcal{Y}, \mathbb{Q})$ gives the existence of $\phi_{s}^{L} \in$ $L^{2}(\Omega \times[0, T], \tilde{\mathbb{Q}} \otimes d\langle P\rangle)$ such that

$$
\begin{aligned}
H & =E_{\tilde{\mathbb{Q}}}\left(H \mid \mathcal{Y}_{0}\right)+\int_{0}^{T} \phi_{s}^{L} d P_{s} \\
& =E_{\tilde{\mathbb{Q}}}(H \mid \sigma(L))+\int_{0}^{T} \phi_{s}^{L} d P_{s} .
\end{aligned}
$$

Remark 3.4 This decomposition does not depend on the choice of the risk neutral probability $\tilde{\mathbb{Q}} \in \mathcal{Q}$ from the informed agent's point of view. Indeed, according to Grorud and Pontier $[20]$, if $\tilde{\mathbb{Q}}_{1}$ and $\tilde{\mathbb{Q}}_{2}$ are two risk neutral probabilities in $\mathcal{Q}$, then $\tilde{\mathbb{Q}}_{1}=f(L) \tilde{\mathbb{Q}}_{2}$, where $f(L)$ is a nonnegative random variable, with mean 1 under $\tilde{\mathbb{Q}}_{2}$. One can then write :

$$
\begin{aligned}
E_{\tilde{\mathbb{Q}}_{1}}(H \mid \sigma(L)) & =\frac{E_{\tilde{\mathbb{Q}}_{2}}(f(L) H \mid \sigma(L))}{E_{\tilde{\mathbb{Q}}_{2}}(f(L) \mid \sigma(L))} \\
& =\frac{f(L) E_{\tilde{\mathbb{Q}}_{2}}(H \mid \sigma(L))}{f(L)} \\
& =E_{\tilde{\mathbb{Q}}_{2}}(H \mid \sigma(L)) .
\end{aligned}
$$

Hence, $E_{\tilde{\mathbb{Q}}}(H \mid \sigma(L))$ does not depend on the choice of $\tilde{\mathbb{Q}}$ risk neutral probability in $\mathcal{Q}$. Then, $\phi_{s}^{L}$ does not either depend on the considered risk-neutral probability measure in $\mathcal{Q}$.

$H$ represents the contingent claim hedged by the informed agent, and $\phi_{s}^{L}=\pi_{s}^{L}$ the unique $\mathcal{Y}$-adapted hedging strategy of the informed agent (see EyraudLoisel [13]). In this continuous framework, all martingales are locally square integrable. So the unique Kunita-Watanabe decomposition of $H$ w.r.t. the $(\tilde{\mathcal{F}}, \tilde{\mathbb{Q}})$-martingale $P$, for any $\tilde{\mathbb{Q}} \in \mathcal{Q}_{N}$, is the following:

$$
H=E_{\tilde{\mathbb{Q}}}(H)+\int_{0}^{T} \phi_{s}^{\tilde{\mathbb{Q}}} d P_{s}+L_{T}^{\tilde{\mathbb{Q}}} \text {. }
$$


Moreover,

$$
V_{t}:=E_{\tilde{\mathbb{Q}}}\left(H \mid \tilde{\mathcal{F}}_{t}\right)=E_{\tilde{\mathbb{Q}}}(H)+\int_{0}^{t} \phi_{s}^{\tilde{\mathbb{Q}}} d P_{s}+L_{t}^{\tilde{\mathbb{Q}}}
$$

where $\phi_{s}^{\tilde{\mathbb{Q}}}$ is $\tilde{\mathcal{F}}_{s}$-measurable and belongs to $L^{2}(\Omega \times[0, T], \tilde{\mathbb{Q}} \otimes d\langle P\rangle)$ and where the rest $\left(L_{t}^{\tilde{\mathbb{Q}}}\right)$ is a $(\tilde{\mathcal{F}}, \tilde{\mathbb{Q}})$-local martingale orthogonal to the stable space generated by $P$, such that $E_{\tilde{\mathbb{Q}}}\left(L_{t}^{\tilde{\mathbb{Q}}}\right)=0$.

We can derive the expression of the integrand (see [15]):

$$
\phi_{s}^{\tilde{\mathbb{Q}}}=\frac{d<V, P>_{s}}{d<P>_{s}}
$$

This expression is not easily exploitable in our case. By using filtering Theory, we derive another expression in terms of projection on the space of all $\tilde{\mathcal{F}}$ adapted processes under any risk neutral probability measure $\tilde{\mathbb{Q}} \in \mathcal{Q}$.

Theorem 3.5 Under any equivalent martingale measure $\tilde{\mathbb{Q}} \in \mathcal{Q}$, the KunitaWatanabe decomposition of $H$ w.r.t. $(\tilde{\mathcal{F}}, \tilde{\mathbb{Q}})$ and process $P$ is the following:

$$
H=E_{\tilde{\mathbb{Q}}}[H]+\int_{0}^{T} E_{\tilde{\mathbb{Q}}}\left(\phi_{s}^{L} \mid \tilde{\mathcal{F}}_{s}\right) d P_{s}+L_{T}^{\tilde{\mathbb{Q}}} \quad a . s .
$$

or in other terms

$$
\phi_{s}^{\tilde{\mathbb{Q}}}=E_{\tilde{\mathbb{Q}}}\left(\phi_{s}^{L} \mid \tilde{\mathcal{F}}_{s}\right)
$$

Proof If $E_{\tilde{\mathbb{Q}}}\left(\phi_{s}^{L} \mid \tilde{\mathcal{F}}_{s}\right)$ satisfies hypothesis of Kunita-Watanabe decomposition given in Definition 3.2, by uniqueness of this decomposition, it is $\phi_{s}^{\tilde{\mathbb{Q}}}$.

First of all, $\left(E_{\tilde{\mathbb{Q}}}\left(\phi_{s}^{L} \mid \tilde{\mathcal{F}}_{s}\right)\right)_{0 \leq s \leq T}$ is $\tilde{\mathcal{F}}_{\text {-adapted. Define : }}$

$$
L_{t}=E_{\tilde{\mathbb{Q}}}\left(H \mid \tilde{\mathcal{F}}_{t}\right)-E_{\tilde{\mathbb{Q}}}(H)-\int_{0}^{t} E_{\tilde{\mathbb{Q}}}\left(\phi_{s}^{L} \mid \tilde{\mathcal{F}}_{s}\right) d P_{s}
$$

It is sufficient to prove that $L$ is a $(\tilde{\mathcal{F}}, \tilde{\mathbb{Q}})$-martingale with mean 0 , orthogonal to $P . \quad L$ is indeed a $(\tilde{\mathcal{F}}, \tilde{\mathbb{Q}})$-martingale: $P$ is a $(\tilde{\mathcal{F}}, \tilde{\mathbb{Q}})$-martingale, so $\int_{0}^{t} E_{\tilde{\mathbb{Q}}}\left(\phi_{s}^{L} \mid \tilde{\mathcal{F}}_{s}\right) d P_{s}$ is a $(\tilde{\mathcal{F}}, \tilde{\mathbb{Q}})$-martingale as integral of a $\tilde{\mathcal{F}}_{\text {-adapted square }}$ integrable process against a $(\tilde{\mathcal{F}}, \tilde{\mathbb{Q}})$-martingale, and $E_{\tilde{\mathbb{Q}}}\left(H \mid \tilde{\mathcal{F}}_{t}\right)$ is a $(\tilde{\mathcal{F}}, \tilde{\mathbb{Q}})$ martingale. Moreover,

$$
E_{\tilde{\mathbb{Q}}}\left(L_{t}\right)=E_{\tilde{\mathbb{Q}}}(H)-E_{\tilde{\mathbb{Q}}}(H)-E_{\tilde{\mathbb{Q}}}\left(\int_{0}^{t} E_{\tilde{\mathbb{Q}}}\left(\phi_{s}^{L} \mid \tilde{\mathcal{F}}_{s}\right) d P_{s}\right)=0
$$

the last term being the expectation of a $(\tilde{\mathcal{F}}, \tilde{\mathbb{Q}})$-martingale null in 0 .

It remains to prove that $L$ is orthogonal to all martingales generated by $d P$, 
stable space generated by $P$, denoted by $\mathcal{M}(d P)$. Using decomposition (3.3) of $H$ in $(\mathcal{Y}, \tilde{\mathbb{Q}})$, we obtain:

$$
\begin{aligned}
L_{t} & =E_{\tilde{\mathbb{Q}}}\left(E_{\tilde{\mathbb{Q}}}(H \mid L)+\int_{0}^{T} \phi_{s}^{L} d P_{s} \mid \tilde{\mathcal{F}}_{t}\right)-E_{\tilde{\mathbb{Q}}}(H)-\int_{0}^{t} E_{\tilde{\mathbb{Q}}}\left(\phi_{s}^{L} \mid \tilde{\mathcal{F}}_{s}\right) d P_{s} \\
= & E_{\tilde{\mathbb{Q}}}\left[\int_{0}^{T} \phi_{s}^{L} d P_{s} \mid \tilde{\mathcal{F}}_{t}\right]-\int_{0}^{t} E_{\tilde{\mathbb{Q}}}\left[\phi_{s}^{L} \mid \tilde{\mathcal{F}}_{s}\right] d P_{s} \\
& +\underbrace{E_{\tilde{\mathbb{Q}}}\left(E_{\tilde{\mathbb{Q}}}(H \mid \sigma(L)) \mid \tilde{\mathcal{F}}_{t}\right)-E_{\tilde{\mathbb{Q}}}(H)}_{N_{t}}
\end{aligned}
$$

where the last term $N_{t}$ is $\tilde{\mathcal{F}}_{t}$-measurable.

The following lemma is a classical result of Filtering Theory (see for example the Saint Flour summer school in Probability Theory course by Pardoux in 1989 on non linear filtering theory [29]).

Lemma 3.6 (Pardoux (1989) [29])

Let $M$ be a $(\mathcal{F}, \tilde{\mathbb{Q}})$-martingale, $\theta \in L_{\text {loc }}^{2}(M)$ on the filtered probability space $(\Omega, \mathcal{Y}, \tilde{\mathbb{Q}})$, such that $\mathcal{F}$ satisfied $\mathcal{F}_{t} \subset \mathcal{Y}_{t} \forall t \in[0, T]$. Then we have:

$$
E_{\tilde{\mathbb{Q}}}\left(\int_{0}^{t} \theta_{s} d M_{s} \mid \mathcal{F}_{t}\right)=\int_{0}^{t} E_{\tilde{\mathbb{Q}}}\left(\theta_{s} \mid \mathcal{F}_{s}\right) d M_{s}
$$

As a consequence of the lemma, we can write

$$
E_{\tilde{\mathbb{Q}}}\left(\int_{0}^{t} \phi_{s}^{L} d P_{s} \mid \tilde{\mathcal{F}}_{t}\right)=\int_{0}^{t} E_{\tilde{\mathbb{Q}}}\left(\phi_{s}^{L} \mid \tilde{\mathcal{F}}_{s}\right) d P_{s}
$$

Using the law of iterated expectations, together with the previous lemma, we can simplify the expression of $L_{t}$ in Equation (3.6):

$$
\begin{aligned}
L_{t} & =E_{\tilde{\mathbb{Q}}}\left[\int_{0}^{T} \phi_{s}^{L} d P_{s} \mid \tilde{\mathcal{F}}_{t}\right]-\int_{0}^{t} E_{\tilde{\mathbb{Q}}}\left(\phi_{s}^{L} \mid \tilde{\mathcal{F}}_{s}\right) d P_{s}+N_{t} \\
& =E_{\tilde{\mathbb{Q}}}\left[E_{\tilde{\mathbb{Q}}}\left[\int_{0}^{T} \phi_{s}^{L} d P_{s} \mid \tilde{\mathcal{F}}_{T}\right] \mid \tilde{\mathcal{F}}_{t}\right]-\int_{0}^{t} E_{\tilde{\mathbb{Q}}}\left(\phi_{s}^{L} \mid \tilde{\mathcal{F}}_{s}\right) d P_{s}+N_{t} \\
& =E_{\tilde{\mathbb{Q}}}\left[\int_{0}^{T} E_{\tilde{\mathbb{Q}}}\left(\phi_{s}^{L} \mid \tilde{\mathcal{F}}_{s}\right) d P_{s} \mid \tilde{\mathcal{F}}_{t}\right]-\int_{0}^{t} E_{\tilde{\mathbb{Q}}}\left(\phi_{s}^{L} \mid \tilde{\mathcal{F}}_{s}\right) d P_{s}+N_{t} \\
& =\int_{0}^{t} E_{\tilde{\mathbb{Q}}}\left(\phi_{s}^{L} \mid \tilde{\mathcal{F}}_{s}\right) d P_{s}-\int_{0}^{t} E_{\tilde{\mathbb{Q}}}\left(\phi_{s}^{L} \mid \tilde{\mathcal{F}}_{s}\right) d P_{s}+N_{t} \\
& =N_{t} .
\end{aligned}
$$

The third equality is obtained because $\int_{0}^{\cdot} E_{\tilde{\mathbb{Q}}}\left(\phi_{s}^{L} \mid \tilde{\mathcal{F}}_{s}\right) d P_{s}$ is a $\left(\tilde{\mathcal{F}}_{t}, \tilde{\mathbb{Q}}\right)$-martingale.

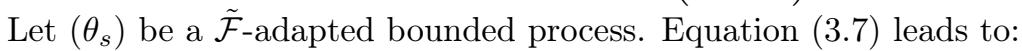

$$
E_{\tilde{\mathbb{Q}}}\left(L_{t} \int_{0}^{t} \theta_{s} d P_{s}\right)=E_{\tilde{\mathbb{Q}}}\left(N_{t} \int_{0}^{t} \theta_{s} d P_{s}\right) .
$$


Recall that $N_{t}$ may be written as:

$$
N_{t}=E_{\tilde{\mathbb{Q}}}(\underbrace{E_{\tilde{\mathbb{Q}}}(H \mid \sigma(L))}_{f(L)} \mid \tilde{\mathcal{F}}_{t})-E_{\tilde{\mathbb{Q}}}(H) .
$$

As $E_{\tilde{\mathbb{Q}}}(H \mid \sigma(L))$ is measurable with respect to the $\sigma$-algebra generated by $L$, it may be written as $f(L)$, where $f$ is a measurable function. Hence :

$$
\begin{aligned}
& E_{\tilde{\mathbb{Q}}}\left[N_{t} \int_{0}^{t} \theta_{s} d P_{s}\right]=E_{\tilde{\mathbb{Q}}}\left[\left[E_{\tilde{\mathbb{Q}}}\left(f(L) \mid \tilde{\mathcal{F}}_{t}\right)-E_{\tilde{\mathbb{Q}}}(H)\right] \int_{0}^{t} \theta_{s} d P_{s}\right] \\
& =E_{\tilde{\mathbb{Q}}}\left(E_{\tilde{\mathbb{Q}}}\left(f(L) \int_{0}^{t} \theta_{s} d P_{s} \mid \tilde{\mathcal{F}}_{t}\right)\right)-E_{\tilde{\mathbb{Q}}}(H) E_{\tilde{\mathbb{Q}}}\left(\int_{0}^{t} \theta_{s} d P_{s}\right) \\
& =E_{\tilde{\mathbb{Q}}}\left(f(L) \int_{0}^{t} \theta_{s} d P_{s}\right) .
\end{aligned}
$$

Literature on initial enlargement of filtration (see Grorud and Pontier [20]) has already established that if there are two risk neutral probability measures $\mathbb{Q}_{1}$ and $\mathbb{Q}_{2}$, then there exists $z$ measurable such that $\mathbb{Q}_{2}=z(L) \mathbb{Q}_{1}$, where $z(L)$ is a $\mathcal{Y}_{0}$-measurable nonnegative random variable, with expectation 1 under $\mathbb{Q}_{1}$, and conversely any such probability measure is risk neutral. For this reason, the previous computations do not depend on the choice of the risk neutral probability measure $\tilde{\mathbb{Q}} \in \mathcal{Q}$. Then let $\mathbb{Q}^{*}$ be the probability measure defined as followed:

$$
\mathbb{Q}^{*}=\frac{f(L)}{E_{\tilde{\mathbb{Q}}}(f(L))} \tilde{\mathbb{Q}} \in \mathcal{Q} .
$$

As $\mathbb{Q}^{*}$ is a risk neutral probability measure, from the previous argument, $\int_{0}^{t} \theta_{s} d P_{s}$ is a $\tilde{\mathcal{F}}$-martingale under $\mathbb{Q}^{*}$. We get

$$
0=E_{\mathbb{Q}^{*}}\left(\int_{0}^{t} \theta_{s} d P_{s}\right)=\frac{1}{E_{\tilde{\mathbb{Q}}}(f(L))} E_{\tilde{\mathbb{Q}}}\left(f(L) \int_{0}^{t} \theta_{s} d P_{s}\right)
$$

We deduce

$$
E_{\tilde{\mathbb{Q}}}\left(f(L) \int_{0}^{t} \theta_{s} d P_{s}\right)=0
$$

and we have finally

$$
E_{\tilde{\mathbb{Q}}}\left[N_{t} \int_{0}^{t} \theta_{s} d P_{s}\right]=0, \forall \theta \tilde{\mathcal{F}}-\text { measurable. }
$$

Under any equivalent martingale measure $\tilde{\mathbb{Q}} \in \mathcal{Q}$, we have the expected orthogonality. This proves that the Kunita-Watanabe decomposition of $H$ has integrand $E_{\tilde{\mathbb{Q}}}\left(\phi_{s}^{L} \mid \tilde{\mathcal{F}}_{s}\right)$ under any risk-neutral probability measure $\tilde{\mathbb{Q}}$ in $\mathcal{Q}$, which ends the proof of Theorem 3.5. 
Remark 3.7 As this integrand determines the unique Kunita-Watanabe decomposition, and as it is the same under any risk-neutral probability measure of $\mathcal{Q}$, then the orthogonal rest is the same and the quadratic residual risk is the same : it may be interpreted as the intrinsic risk of the contingent claim $H$.

Remark 3.8 This also proves that $E_{\tilde{\mathbb{Q}}}\left(\phi_{s}^{L} \mid \tilde{\mathcal{F}}_{s}\right)$ does not depend on the choice of $\tilde{\mathbb{Q}} \in \mathcal{Q}$. This can also be proved by the following way :

Let $Y_{s} \in \tilde{\mathcal{F}}_{s}, Q_{2}=z(L) Q_{1}$, from the previous argument, it follows that

$$
E_{Q_{1}}\left[Y_{s} E_{Q_{2}}\left[\phi_{s}^{L} / \tilde{\mathcal{F}}_{s}\right] E_{Q_{1}}\left[z(L) / \tilde{\mathcal{F}}_{s}\right]\right]=E_{Q_{1}}\left[Y_{s} \phi^{L} z(L)\right] .
$$

The main consequence of the previous Theorem is then:

Proposition 3.9 Prices are the same under two different risk-neutral probability measures.

Remark 3.10 We obtain here a result which is coherent with the result of Grorud and Pontier [20] in a different framework, according to whom all riskneutral probability measures in an incomplete market (where incompleteness is due to additional information) give the same market price.

Remark 3.11 Our study is also coherent with Föllmer and Schweizer's work in [15], but several points differ: their hypothesis of invariance of the decomposition of Doob-Meyer of the prices does not remain valid in our case. They suppose that the decomposition of Doob-Meyer of the prices under filtration $\tilde{\mathcal{F}}$ and probability $\mathbb{Q}, P=P_{0}+M+A$ where $A$ is $\tilde{\mathcal{F}}$-predictable, and where $M$ is a $(\tilde{\mathcal{F}}, \mathbb{Q})$-martingale, stays the decomposition of Doob-Meyer of the prices in the larger filtration, in other words, $M$ remain a $(\mathcal{Y}, \mathbb{Q})$-martingale, although adapted to $\tilde{\mathcal{F}}$ (See Hypotheses (4.1) to (4.3) in [15]). These hypotheses, although weaker, are similar to the so-called Hypothesis $(\mathbf{H})$ in Filtering Theory (used also in progressive enlargement of filtration, in models as those of Jeanblanc $[24,4])$. Instead of such hypothesis, our study takes place under Hypothesis $\left(\mathbf{H}_{\mathbf{3}}\right)$. Furthermore, we distinguish here three different filtrations: the filtration of the Brownian motion, the filtration of prices, and the filtration enlarged with the information. On the other hand, as pointed out before, our study joins perfectly within the framework of incompleteness that they studied: the incompleteness due to a lack of information.

Finally, our results are coherent with those in previous literature, whereas the model is a bit different. The present study illustrates in a different framework the same kind of incompleteness already studied in this literature: incompleteness due to a lack of information.

\subsection{Clark-Ocone Formula}

Since $\phi_{s}^{\tilde{Q}}$ may be expressed from $\phi_{s}^{L}$, it is important to study how to obtain $\phi_{s}^{L}$. We express it as a function of the Malliavin derivative of $H$, via the use of 
a Clark-Ocone Formula. We can not use directly the standard formula in our case, under enlarged filtration $\mathcal{Y}$, as $\mathcal{Y}_{0}=\sigma(L)$ is not trivial. Nevertheless, thanks to a transformation and the independence of $\sigma(L)$ and $\mathcal{F}$ under $\mathbb{Q}$, we will use the existing Clark-Ocone Formula under filtration $\mathcal{F}$, and obtain an expression of $\phi_{s}^{L}$.

Theorem 3.12 If $H \in L^{2}(\Omega, \mathcal{Y}, Q)$ and $\forall x, H(., x) \in \mathbb{D}^{1,2}$, we have

$$
\phi_{s}^{L}=\left(\left.E_{\mathbb{Q}}\left[D_{s}(H(., x)) \mid \mathcal{F}_{s}\right]\right|_{x=L}\right)\left(\sigma_{s}^{L}\right)^{-1} .
$$

Proof In the enlarged space $\mathcal{Y}$, under $\left(\mathbf{H}_{\mathbf{3}}\right), \sigma(L)$ and $\mathcal{F}_{t}$ are independent. Moreover, $\left.\mathbb{Q}\right|_{\mathcal{F}}=\mathbb{P}$ and $\left.\mathbb{Q}\right|_{\sigma(L)}=\mathbb{P}^{L}$ (law of $L$ ).

$H$ is measurable w.r.t. $\mathcal{Y}_{T}$, so $H$ may be written as $H=H(W, L)$ (thanks to the independence and because $\mathcal{Y}_{T}$ is generated by $\mathcal{F}$ and $\left.\sigma(L)\right)$. Then, at fixed $L=x, H(W, x)$ is $\mathcal{F}_{T}$-measurable. So its representation is given by the standard Clark-Ocone Formula:

$$
\begin{aligned}
H(W, x) & =E_{\mathbb{P}}(H(W, x))+\int_{0}^{T} E_{\mathbb{P}}\left(D_{s}(H(W, x)) \mid \mathcal{F}_{s}\right) d W_{s} \quad \mathbb{P} \text { a.s. } \\
& =\left.E_{\mathbb{Q}}(H \mid L)\right|_{L=x}+\int_{0}^{T} E_{\mathbb{Q}}\left(D_{s}(H(W, x)) \mid \mathcal{F}_{s}\right) d W_{s} .
\end{aligned}
$$

Indeed, derive $H$ w.r.t. $W$ with $L=x$ has a sense under $\mathbb{Q}$ as $W$ and $L$ are independent, and derive $W \mapsto H(W, L)$ under $\mathbb{Q}$ or $\mathbb{P}$ is the same, as $W$ is a $\mathbb{P}$ and also a $\mathbb{Q}$-Brownian motion. Again using independence between $\sigma(L)$ and $\mathcal{F}_{t}$, we can write

$$
H(W, L)=\left.H(W, x)\right|_{x=L}
$$

and an identification leads to:

$$
\sigma_{s}^{L} \phi_{s}^{L}=\left.E_{\mathbb{Q}}\left[D_{s}(H(W, x)) \mid \mathcal{F}_{s}\right]\right|_{x=L} .
$$

Equation (3.8) directly comes from Equation (3.9) and the fact that $\sigma_{s}^{L}$ is invertible, which ends the proof of Theorem 3.12 .

\subsection{Residual Risk}

3.4.1 Expression of the residual risk under a risk neutral probability in $\mathcal{Q}_{N}$

We derivean expression of the intrinsic residual risk of the contingent claim $H$ under a risk neutral probability in $\mathcal{Q}_{N}$.

Proposition 3.13 Let $\mathbb{Q}^{*} \in \mathcal{Q}_{N}$.

The residual risk $L_{t}^{\mathbb{Q}^{*}}$ has the following expression:

$$
L_{t}^{\mathbb{Q}^{*}}=\int_{0}^{t}\left[E_{\mathbb{Q}^{*}}\left(\phi_{s}^{L} \mid \tilde{\mathcal{F}}_{s}\right)-\phi_{s}^{\mathbb{Q}^{*}}\right] d P_{s}+E_{\mathbb{Q}^{*}}\left[E_{\mathbb{Q}^{*}}(H \mid \sigma(L)) \mid \tilde{\mathcal{F}}_{t}\right]-E_{\mathbb{Q}^{*}}(H) .
$$


A measure of the risk of detaining the contingent claim $H$ in this incomplete market is given by the variance of $L_{T}^{\mathbb{Q}^{*}}$ at terminal time:

$$
\begin{aligned}
\operatorname{Var}_{\mathbb{Q}^{*}}\left(L_{T}^{\mathbb{Q}^{*}}\right)= & E_{\mathbb{Q}^{*}}\left(\int_{0}^{T}\left[E_{\mathbb{Q}^{*}}\left(\phi_{s}^{L} \mid \tilde{\mathcal{F}}_{s}\right)-\phi_{s}^{\mathbb{Q}^{*}}\right] d P_{s} \times\left(E_{\mathbb{Q}^{*}}\left[E_{\mathbb{Q}^{*}}(H \mid \sigma(L)) \mid \tilde{\mathcal{F}}_{T}\right]-E_{\mathbb{Q}^{*}}(H)\right)\right) \\
& +E_{\mathbb{Q}^{*}}\left(\left(E_{\mathbb{Q}^{*}}\left[E_{\mathbb{Q}^{*}}(H \mid \sigma(L)) \mid \tilde{\mathcal{F}}_{T}\right]-E_{\mathbb{Q}^{*}}(H)\right)^{2}\right) .
\end{aligned}
$$

Proof Let $\mathbb{Q}^{*} \in \mathcal{Q}_{N}$ a risk neutral probability measure for the non informed agent. The expression of the residual risk $L_{t}^{\mathbb{Q}^{*}}$ at time $t$ is given by Equations (3.4) and (3.5), which are true under any $\mathbb{Q}^{*} \in \mathcal{Q}_{N}$ :

$L_{t}^{\mathbb{Q}^{*}}=E_{\mathbb{Q}^{*}}\left[\int_{0}^{T} \phi_{s}^{L} d P_{s} \mid \tilde{\mathcal{F}}_{t}\right]-\int_{0}^{t} \phi_{s}^{\mathbb{Q}^{*}} d P_{s}+E_{\mathbb{Q}^{*}}\left[E_{\mathbb{Q}^{*}}(H \mid \sigma(L)) \mid \tilde{\mathcal{F}}_{t}\right]-E_{\mathbb{Q}^{*}}(H)$.

The second part of this expression corresponds to the difference of prices of the contingent claim under $\mathbb{Q}^{*}$, whether the agent has or does not have the additional information, which is the difference of prices on the informed (complete) market and the non informed (incomplete) market. In the first case, the price will be $E_{\mathbb{Q}^{*}}(H \mid \sigma(L))$ projected on the $\tilde{\mathcal{F}}_{t}$-measurable random variables (which means attainable prices), and in the other case, the price is $E_{\mathbb{Q}^{*}}(H)$. The first part may be rewritten thanks to Lemma 3.6:

$$
\begin{aligned}
E_{\mathbb{Q}^{*}}\left[\int_{0}^{T} \phi_{s}^{L} d P_{s} \mid \tilde{\mathcal{F}}_{t}\right] & =E_{\mathbb{Q}^{*}}\left[E_{\mathbb{Q}^{*}}\left(\int_{0}^{T} \phi_{s}^{L} d P_{s} \mid \tilde{\mathcal{F}}_{T}\right) \mid \tilde{\mathcal{F}}_{t}\right] \\
& =E_{\mathbb{Q}^{*}}\left[\int_{0}^{T} E_{\mathbb{Q}^{*}}\left(\phi_{s}^{L} \mid \tilde{\mathcal{F}}_{s}\right) d P_{s} \mid \tilde{\mathcal{F}}_{t}\right] \\
& =\int_{0}^{t} E_{\mathbb{Q}^{*}}\left(\phi_{s}^{L} \mid \tilde{\mathcal{F}}_{s}\right) d P_{s},
\end{aligned}
$$

as $P$ is a $\left(\tilde{\mathcal{F}}, \mathbb{Q}^{*}\right)$-martingale.

The residual risk may be rewritten as:

$$
L_{t}^{\mathbb{Q}^{*}}=\int_{0}^{t}\left[E_{\mathbb{Q}^{*}}\left(\phi_{s}^{L} \mid \tilde{\mathcal{F}}_{s}\right)-\phi_{s}^{\mathbb{Q}^{*}}\right] d P_{s}+E_{\mathbb{Q}^{*}}\left[E_{\mathbb{Q}^{*}}(H \mid \sigma(L)) \mid \tilde{\mathcal{F}}_{t}\right]-E_{\mathbb{Q}^{*}}(H) .
$$

The sum of these two terms forms the residual risk due to the lack of information of the non informed agent. This risk is the minimal risk under $\mathbb{Q}^{*}$ which the non informed agent can hope to take by holding the option (see Föllmer and Schweizer [15] and Pham [30]). It is the constituent of the option which is orthogonal to the market prices (see Kunita-Watanabe decomposition).

A measure of this risk, represented by $L_{t}^{\mathbb{Q}^{*}}$, martingale with null expectation, is its variance $\operatorname{Var}_{\mathbb{Q}^{*}}\left(L_{T}^{\mathbb{Q}^{*}}\right)$, which represents the quadratic residual risk at 
terminal time $T$.

At terminal time, we can write:

$$
L_{T}^{\mathbb{Q}^{*}}=\int_{0}^{T}\left[E_{\mathbb{Q}^{*}}\left(\phi_{s}^{L} \mid \tilde{\mathcal{F}}_{s}\right)-\phi_{s}^{\mathbb{Q}^{*}}\right] d P_{s}+E_{\mathbb{Q}^{*}}\left[E_{\mathbb{Q}^{*}}(H \mid \sigma(L)) \mid \tilde{\mathcal{F}}_{T}\right]-E_{\mathbb{Q}^{*}}(H) .
$$

So

$$
\begin{aligned}
\operatorname{Var}_{\mathbb{Q}^{*}}\left(L_{T}^{\mathbb{Q}^{*}}\right)= & E_{\mathbb{Q}^{*}}\left(\left(\int_{0}^{T}\left[E_{\mathbb{Q}^{*}}\left(\phi_{s}^{L} \mid \tilde{\mathcal{F}}_{s}\right)-\phi_{s}^{\mathbb{Q}^{*}}\right] d P_{s}\right)^{2}\right) \\
& +2 E_{\mathbb{Q}^{*}}\left(\int_{0}^{T}\left[E_{\mathbb{Q}^{*}}\left(\phi_{s}^{L} \mid \tilde{\mathcal{F}}_{s}\right)-\phi_{s}^{\mathbb{Q}^{*}}\right] d P_{s} \times\left(E_{\mathbb{Q}^{*}}\left[E_{\mathbb{Q}^{*}}(H \mid \sigma(L)) \mid \tilde{\mathcal{F}}_{T}\right]-E_{\mathbb{Q}^{*}}(H)\right)\right) \\
& +E_{\mathbb{Q}^{*}}\left(\left(E_{\mathbb{Q}^{*}}\left[E_{\mathbb{Q}^{*}}(H \mid \sigma(L)) \mid \tilde{\mathcal{F}}_{T}\right]-E_{\mathbb{Q}^{*}}(H)\right)^{2}\right) .
\end{aligned}
$$

As $L_{T}^{\mathbb{Q}^{*}}$ is orthogonal to $P$, this expression may be slightly simplified, and the variance may be rewritten as:

$$
\begin{aligned}
\operatorname{Var}_{\mathbb{Q}^{*}}\left(L_{T}^{\mathbb{Q}^{*}}\right)= & E_{\mathbb{Q}^{*}}\left(\int_{0}^{T}\left[E_{\mathbb{Q}^{*}}\left(\phi_{s}^{L} \mid \tilde{\mathcal{F}}_{s}\right)-\phi_{s}^{\mathbb{Q}^{*}}\right] d P_{s} \times\left(E_{\mathbb{Q}^{*}}\left[E_{\mathbb{Q}^{*}}(H \mid \sigma(L)) \mid \tilde{\mathcal{F}}_{T}\right]-E_{\mathbb{Q}^{*}}(H)\right)\right) \\
& +E_{\mathbb{Q}^{*}}\left(\left(E_{\mathbb{Q}^{*}}\left[E_{\mathbb{Q}^{*}}(H \mid \sigma(L)) \mid \tilde{\mathcal{F}}_{T}\right]-E_{\mathbb{Q}^{*}}(H)\right)^{2}\right) .
\end{aligned}
$$

This is the general expression of the quadratic residual risk under a probability measure in $\mathcal{Q}_{N}$.

In the case of a measure in $\mathcal{Q}_{N}$ but not in $\mathcal{Q}$, we can not simplify better the previous expression. Nevertheless, with orthogonality arguments we obtain a simpler expression.

Proposition 3.14 A measure of the residual risk under a risk neutral probability measure $\mathbb{Q}^{*} \in \mathcal{Q}_{N} \backslash \mathcal{Q}$ is given by:

$$
\operatorname{Var}_{\mathbb{Q}^{*}}\left(L_{T}^{\mathbb{Q}^{*}}\right)=E_{\mathbb{Q}^{*}}\left(\left(H-E_{\mathbb{Q}^{*}}(H)\right)^{2}\right)-E_{\mathbb{Q}^{*}}\left(\left(\int_{0}^{T} \phi_{s}^{\mathbb{Q}^{*}} d P_{s}\right)^{2}\right) .
$$

Proof Let $\mathbb{Q}^{*} \in \mathcal{Q}_{N}$ be a probability measure that does not belong to $\mathcal{Q}$. Then the Kunita-Watanabe decomposition may be written as follows:

$$
H-E_{\mathbb{Q}^{*}}(H)=\int_{0}^{T} \phi_{s}^{\mathbb{Q}^{*}} d P_{s}+L_{T}^{\mathbb{Q}^{*}},
$$

$P$ being a $\left(\tilde{\mathcal{F}}, \mathbb{Q}^{*}\right)$-martingale, but not a $\left(\mathcal{Y}, \mathbb{Q}^{*}\right)$-martingale (otherwise $\mathbb{Q}^{*}$ would belong to $\mathcal{Q}$ ). There is no martingale representation Theorem.

As $L_{T}^{\mathbb{Q}^{*}}$ is orthogonal to the price process, we get Equation (3.12) and the expected expression of the quadratic residual risk. 


\subsubsection{Expression of the residual risk under a risk neutral probability in $\mathcal{Q}$}

In the case of a measure in $\mathcal{Q} \subset \mathcal{Q}_{N}$, Equations (3.10) and (3.12) of Proposition ?? may be simplified. We obtain the following result:

Proposition 3.15 A measure of the residual risk under a risk neutral probability measure $\mathbb{Q}^{*}$ in $\mathcal{Q}$ is:

$$
\operatorname{Var}\left(L_{T}^{\mathbb{Q}^{*}}\right)=E_{\mathbb{Q}^{*}}\left(E_{\mathbb{Q}^{*}}\left[E_{\mathbb{Q}^{*}}(H \mid \sigma(L)) \mid \tilde{\mathcal{F}}_{T}\right]^{2}\right)-E_{\mathbb{Q}^{*}}(H)^{2},
$$

Proof In the case of a risk neutral probability $\mathbb{Q}^{*}$ in $\mathcal{Q}$, the first term of $L_{T}^{\mathbb{Q}^{*}}$ in Equation (3.10) nullifies, in application of Proposition 3.5. The expression becomes simpler, and we deduce Equation (3.13), which gives a measure of the residual risk under a risk neutral probability $\mathbb{Q}^{*} \in \mathcal{Q}$.

Remark 3.16 $\operatorname{Var}\left(L_{T}^{\mathbb{Q}^{*}}\right)$ measures the revelation of the information in the prices, because the not informed agent does not possess the whole information, but she "sees" all the same a part of the information showing through in market prices.

A minimal risk exists, which is taken over all possible equivalent martingale measures in $\mathcal{Q}$ and it is the following:

$$
\inf _{Q^{*} \in \mathcal{Q}}\left\{E_{\mathbb{Q}^{*}}\left(E_{\mathbb{Q}^{*}}\left[E_{\mathbb{Q}^{*}}(H \mid \sigma(L)) \mid \tilde{\mathcal{F}}_{T}\right]^{2}\right)-E_{\mathbb{Q}^{*}}(H)^{2}\right\} .
$$

It can be approached by a minimizing sequence.

Remark 3.17 We can notice that measuring the residual risk under a riskneutral probability is arbitrary, because choosing this equivalent martingale measure is already an arbitrary choice, and also because at first, the model took into account a historic probability, which we do not take into account in this measure of risk. Moreover the estimated risk is the risk of this model under this measure, and not the intrinsic risk.

3.5 Existence of a minimal martingale measure

Let us write the Doob-Meyer decomposition of the $(\tilde{\mathcal{F}}, \mathbb{Q})$-semi-martingale of prices $P$ :

$$
P_{t}=P_{0}+M_{t}+A_{t},
$$

where $M$ is a $(\tilde{\mathcal{F}}, \mathbb{Q})$-martingale of null expectation (as a uniformly integrable local martingale, from Assumption (A1), see Lépingle and Mémin [28] Theorems II-2 and III-7), and $A$ is a $\tilde{\mathcal{F}}$-predictable finite-variations process, whose increments are square integrable under $\mathbb{Q}$ and satisfy $A_{0}=0$.

By Girsanov Theorem, and as Assumption ( $A 1)$ ensures the existence of an equivalent martingale measure, $A$ may be written as follows:

$$
A_{t}=\int_{0}^{t} \lambda_{u} d<M>_{u}, 0 \leq t \leq T,
$$


where $\lambda$ is a $\tilde{\mathcal{F}}$-predictable process.

Define

$$
K_{t}=\int_{0}^{t} \lambda_{u}^{\prime} d<M>_{u} \lambda_{u}, 0 \leq t \leq T
$$

and denote by $\hat{Z}$ the process solution of the following SDE:

$$
d \hat{Z}_{t}=-\hat{Z}_{t} \lambda_{t} d M_{t}, 0, \leq t \leq T, \quad \hat{Z}_{0}=1 .
$$

Then:

$$
\hat{Z}_{t}=\exp \left(-\int_{0}^{t} \lambda_{u} d M_{u}-\frac{1}{2} K_{t}\right), 0 \leq t \leq T .
$$

$\hat{Z}$ is a positive uniformly integrable $(\tilde{\mathcal{F}}, \mathbb{Q})$-local martingale, so it is a $(\tilde{\mathcal{F}}, \mathbb{Q})$ martingale. This process defines a probability measure $\hat{\mathbb{Q}}$ equivalent to $\mathbb{Q}$ by

$$
\frac{d \hat{\mathbb{Q}}}{d \mathbb{Q}}=\hat{Z}_{T} \in L^{2}(\mathbb{Q}) .
$$

As $P$ is a continuous process and $\hat{Z} \in \mathcal{M}^{2}(\mathbb{Q})$, Proposition 4.3 of Pham [30] may apply, and we establish the following result in our case:

Proposition 3.18 Probability measure $\hat{\mathbb{Q}}$ is an equivalent martingale measure, called minimal martingale measure. It satisfies the following property: any square integrable $(\tilde{\mathcal{F}}, \mathbb{Q})$-martingale orthogonal to $M$ under $\mathbb{Q}$ remains a $(\tilde{\mathcal{F}}, \hat{\mathbb{Q}})$-martingale:

$$
L \in \mathcal{M}^{2}(\tilde{\mathcal{F}}, \mathbb{Q}), L \perp_{\mathbb{Q}} M \Rightarrow L \text { is a martingale under }(\tilde{\mathcal{F}}, \hat{\mathbb{Q}}) .
$$

Also following Pham [30] (Paragraph 4.3 Theorem 4.2), we can mention the following result, which gives an expression of the strategy minimizing the local risk, in the case where $K$ is uniformly bounded:

Proposition 3.19 If $K$ is uniformly bounded, there exists a strategy minimizing the local risk, given by:

$$
\begin{aligned}
V_{t}^{*} & =E_{\hat{\mathbb{Q}}}\left(H \mid \tilde{\mathcal{F}}_{t}\right) \\
\phi_{t}^{*} & =\phi_{t}^{H},
\end{aligned}
$$

where $\phi_{t}^{H}$ is the integrand of the Föllmer-Schweizer decomposition of $H$ under $\mathbb{Q}$, which is:

$$
\begin{aligned}
& H=H_{0}+\int_{0}^{T} \phi_{t}^{H} d P_{t}+L_{t}^{H} \\
& H_{0} \in \mathbb{R}, \phi^{H} \in L^{2}(M) \cap L^{2}(A), L^{H} \in \mathcal{M}(\tilde{\mathcal{F}}, \mathbb{Q}) \perp M .
\end{aligned}
$$

Proof It is the Kunita-Watanabe decomposition of the $(\tilde{\mathcal{F}}, \hat{\mathbb{Q}})$-martingale $V^{*}$ against the $(\tilde{\mathcal{F}}, \hat{\mathbb{Q}})$-martingale $P$. Indeed, $L^{H}$ is a $(\tilde{\mathcal{F}}, \mathbb{Q})$-martingale orthogonal to $M$, and so remains a martingale under $\hat{\mathbb{Q}}$.

Remark 3.20 By uniqueness of the Kunita-Watanabe decomposition under $\hat{\mathbb{Q}}$, we have $H_{0}=E_{\hat{\mathbb{Q}}}(H)$, which is the right term of the Föllmer-Schweizer decomposition. 


\section{Example}

Let us give an example satisfying all hypotheses of the previous model. This example was first introduced in the last section of [13]. Suppose that the price process is driven by the following dynamics (stochastic volatility model) :

$$
d P_{t}=b^{\prime}\left(P_{t}, X_{t}, \pi_{t}\right) P_{t} d t+\sigma_{t}^{\prime}(\eta) P_{t} d W_{t},
$$

where

$$
\sigma_{t}^{\prime}(\eta)=\sigma^{0} \mathcal{I}_{[0, \eta[}(t)+\sigma^{1} \mathcal{I}_{[\eta, T]}(t), \sigma^{0}, \sigma^{1} \neq 0 .
$$

The volatility of this model is piecewise constant, taking two possible values $\sigma^{0}$ and $\sigma^{1}$ fixed by the model, $\eta$ is a random variable satisfying Hypothesis $\left(\mathbf{H}_{3}\right)$, taking its values in $[0, T+\varepsilon]$.

The information of the insider trader is the following strong initial information: $L=\eta, \mathcal{F}_{T+\varepsilon}-$ measurable . The drift parameter is chosen as the following:

$$
b^{\prime}\left(X_{t}, P_{t}, \pi_{t}\right)=b_{0}+\frac{b_{1}}{\left(1+P_{t}\right)\left(1+\pi_{t}^{2}\right)}, \quad b_{0}, b_{1} \in \mathbb{R} \text { fixed. }
$$

Interest rate $r$ is supposed to be constant.

Drift $b^{\prime}$ is bounded, and may vary between two thresholds $b_{0}$ and $b_{0}+a$. Two cases may appear, depending on the sign of $b_{1}$. If $b_{1}<0$, the influence is a positive influence: the bigger is the investment portfolio, the higher is the drift of the prices. This is moderated by the level of prices: the higher are the prices, the lower is the influence. If $b_{1}>0$, it is the converse principle: when the level of the portfolio increases, the drift of the prices decreases, and the influence is stronger when the level of prices is high. Remark that the case $b_{1}=0$ is the case treated in the previous section, without influence.

Depending on the sign of $b_{1}$, representing the amplitude of the influence, this influence will have either a leverage effect or a return effect on the drift of the price process around the value $b_{0}$. The influence is from the insider's portfolio on the price process, which remains bounded according to the hypotheses.

We can also notice that

$$
\sigma_{s}^{\prime-1}\left(b_{s}^{\prime}-r_{s}\right)=\sigma_{s}^{\prime-1}\left(b_{0}-r+\frac{b_{1}}{\left(1+P_{t}\right)\left(1+\pi_{t}^{2}\right)}\right)
$$

is bounded, as well as $\sigma^{\prime}$. So there exists a risk-neutral probability measure $\tilde{\mathbb{Q}}$ under which $d P_{t}=\sigma_{t}^{\prime} P_{t} d \tilde{W}_{t}$ is a positive uniformly integrable martingale.

Remark 4.1 We don't have here constraints on the signs of $b_{0}$ or $b_{1}$, whereas it is often the case in previous influence models developed in the literature, such as in the model introduced by Cuoco and Cvitanic (1998) [9], and treated deeply in Grorud and Pontier (2005) [21] (their influence form is slightly different from the one treated in this work). This may be explained by the fact that we consider a hedging problem, whereas they considered an optimization problem, and therefore we do not need the convexity of the parameters here. 
For the present model, considering the hedging of a European call option with maturity $T$ and strike $K$, the parameters are the following :

$$
\begin{aligned}
f\left(s, P_{s}, X_{s}, \pi_{s}\right) & =X_{s} r+\left(b_{0}-r+\frac{b_{1}}{\left(1+P_{t}\right)\left(1+\pi_{t}^{2}\right)}\right) \pi_{s}, \\
g\left(P_{T}\right) & =\left(P_{T}-K\right)_{+} .
\end{aligned}
$$

This leads to the following FBSDE modeling the hedging problem of the informed agent :

$$
\left\{\begin{array}{l}
P_{t}=P_{0}+\int_{0}^{t}\left(b_{0}+\frac{b_{1}}{\left(1+P_{t}\right)\left(1+\pi_{t}^{2}\right)}\right) P_{s} d s+\int_{0}^{t} \sigma_{s}(\eta) P_{s} d W_{s} \\
X_{t}=\left(P_{T}-K\right)_{+}-\int_{t}^{T}\left(X_{s} r+\left(b_{0}+a h\left(\pi_{s}\right)\right) \pi_{s}-r \pi_{s}\right) d s-\int_{t}^{T} \sigma_{s}(\eta) \pi_{s} d W_{s}
\end{array}\right.
$$

According to [13], hypotheses of Theorem 2.2 are satisfied, and the influent informed agent has a unique hedging strategy in this market. As explained before, the informed market is complete, whereas the non informed market is incomplete.

From Subsection 3.3, we derive an expression of the strategy of the informed agent:

$$
\sigma_{s}^{L} \phi_{s}^{L}=\left.E_{\mathbb{Q}}\left[D_{s}(H(W, x)) \mid \mathcal{F}_{s}\right]\right|_{x=L} .
$$

In the case of a European call option $H(W, x)=\left(P_{T}^{x}-K\right)_{+}$, where $P_{T}^{x}$ is the price at terminal time when the volatility jump appeared at time $x$,

$$
\sigma_{s}^{L} \phi_{s}^{L}=E_{\mathbb{Q}}\left[1_{P_{T}^{x}>K} D_{s} P_{T}^{x} \mid \mathcal{F}_{s}\right]_{x=L}
$$

We obtain an expression of the optimal hedging strategy of a non informed agent in the market, thanks to results of Section 3 (Equation (3.8)):

$$
\phi_{s}^{\mathbb{Q}^{*}}=E_{\mathbb{Q}^{*}}\left[\left(\sigma_{s}^{L}\right)^{-1} E_{\mathbb{Q}}\left[1_{P_{T}^{x}>K} D_{s} P_{T}^{x} \mid \mathcal{F}_{s}\right]_{x=L} \mid \tilde{\mathcal{F}}_{s}\right]
$$

We derive also the expression of the quadratic residual risk from Equation (3.13):

$$
\operatorname{Var}\left(L_{T}^{\mathbb{Q}^{*}}\right)=E_{\mathbb{Q}^{*}}\left(E_{\mathbb{Q}^{*}}\left[E_{\mathbb{Q}^{*}}\left(\left(P_{T}^{L}-K\right)_{+} \mid L\right) \mid \tilde{\mathcal{F}}_{T}\right]^{2}\right)-E_{\mathbb{Q}^{*}}\left(\left(P_{T}^{L}-K\right)_{+}\right)^{2}
$$

Remark 4.2 We have in this example

$$
\sigma_{t}(L)^{2}=\frac{1}{P_{t}^{2}} \frac{d<P>_{t}}{d t}
$$

So $\sigma_{t}(L)^{2}$ is $\tilde{\mathcal{F}}$-measurable, hence observable. 


\section{Conclusion}

As a consequence of the last remark, imagine a weak informed agent, who only knows the law of $L$ (such information is commonly called weak information). Then, as soon as the agent observes the jump in the volatility process, she has the same information as the strong informed agent. This would mean intuitively that in this example, the weak information would be enough to complete the market, because it would be sufficient to hedge against this contingent claim. A possible continuation of this model would be the study of BSDE or FBSDE with other types of additional information (weak, progressive), and thus under other types of enlargement of filtration. Other techniques were proposed to study the presence of asymmetry of information, as Malliavin calculus (Imkeller [22], Nualart et al. [27]), either models of weak information (Baudoin [3]) or models of punters (Corcuera, Imkeller, Kohatsu-Higa and Nualart $[8])$.

Several studies have been developed on discretization schemes for BSDE and FBSDE, from Chevance (1997) [7], to Delarue (2002) [10], Gobet, Lemor and Warin (2005) [17], or more recently Bouchard and Elie (2008) [5], or Bouchard, Elie and Touzi (2009) [6]. Even if these new schemes may open a way to simulate and use more extensively such study, and even if these new works and their future extensions give interesting tracks for efficient schemes for FBSDEs, there is still a difficulty that leaves in the use of such equations. The main difficulty is to express explicitly the solutions, especially when the filtration is not easy to express as an enlarged filtration of the Brownian filtration. Since then, the risk minimization and the quadratic hedging approaches allows to have all the same an expression of the hedging strategy, and of the minimal risk a non informed trader is taking by detaining the contingent claim.

\section{References}

1. J. Amendinger. Initial enlargement of filtrations and additionnal information of financial markets. Doctoral Thesis, 1999.

2. J. P. Ansel and C. Stricker. Décomposition de Kunita-Watanabe. In Séminaire de Probabilités, XXVII, volume 1557 of Lecture Notes in Math., pages 30-32. Springer, Berlin, 1993.

3. F. Baudoin. Modeling anticipations on financial markets. In Paris-Princeton Lectures on Mathematical Finance, 2002, volume 1814 of Lecture Notes in Math., pages 43-94. Springer, Berlin, 2003.

4. C. Blanchet-Scalliet and M. Jeanblanc. Hazard rate for credit risk and hedging defaultable contingent claims. Finance Stoch., 8(1):145-159, 2004.

5. B. Bouchard and R. Elie. Discrete time approximation of decoupled forward-backward sde with jumps. Stoch. Proc. and App., 118(1):53-75, 2008.

6. B. Bouchard, R. Elie, and N. Touzi. Discrete-time approximation of bsdes and probabilistic schemes for fully nonlinear pdes.

7. D. Chevance. Résolution numériques des équations différentielles stochastiques rétrogrades. PhD thesis, Université de Provence - Aix-Marseille I, Marseille, 1997.

8. J.M. Corcuera, P. Imkeller, A. Kohatsu-Higa, and D. Nualart. Additional utility of insiders with imperfect dynamical information. Fin. and Stoch., 8:437-450, 2004. 
9. D. Cuoco and J. Cvitanić. Optimal consumption choices for a "large" investor. J. Econom. Dynam. Control, 22(3):401-436, 1998.

10. F. Delarue. On the existence and uniqueness of solutions to fbsdes in a non-degenerate case. Stochastic Process. Appl., 99(2):209-286, 2002.

11. F. Delbaen and W. Schachermayer. A general version of the fundamental theorem of asset pricing. Mathematische Annalen, 300(3):463-520, 1994.

12. A. Eyraud-Loisel. Backward stochastic differential equations with enlarged filtration. option hedging of an insider trader in a financial market with jumps. Stochastic Processes and their Applications, 115(11):1745-1763, 2005.

13. A. Eyraud-Loisel. Option hedging by an influent informed investor. Working Paper, 2009.

14. A. Eyraud-Loisel. EDSR et EDSPR avec grossissement de filtration, problèmes d'asymétrie d'information et de couverture sur les marchés financiers. PhD thesis, Université Paul Sabatier, Toulouse, December 2005.

15. H. Föllmer and M. Schweizer. Hedging of contingent claims under incomplete information. In Applied stochastic analysis (London, 1989), volume 5 of Stochastics Monogr. pages 389-414. Gordon and Breach, New York, 1991.

16. H. Föllmer and D. Sondermann. Hedging of nonredundant contingent claims. In Contributions to mathematical economics, pages 205-223. North-Holland, Amsterdam, 1986.

17. E. Gobet, J-P. Lemor, and X. Warin. A regression-based monte-carlo method to solve backward stochastic differential equations. To appear in Annals of Applied Probability, 2005.

18. C. Gourieroux, J-P. Laurent, and Huyên P. Mean-variance hedging and numéraire. Math. Finance, 8(3):179-200, 1998

19. A. Grorud and M. Pontier. Insider trading in a continuous time market model. Int. Journal of Theor. and App. Fin., 1(3):331-347, 1998.

20. A. Grorud and M. Pontier. Probabilités neutres au risque et asymétrie d'information. C. R. Acad. Sci. Paris Sér. I Math., 329(1):1009-1014, 1999.

21. A. Grorud and M. Pontier. Fiancial market model with influential informed investors. submitted, 2005.

22. P. Imkeller. Enlargement of the Wiener filtration by an absolutely continuous random variable via Malliavin's calculus. Probab. Theory Related Fields, 106(1):105-135, 1996.

23. J. Jacod and A. N. Shiryaev. Limit theorems for stochastic processes, volume 288 of Grundlehren der Mathematischen Wissenschaften /Fundamental Principles of Mathematical Sciences]. Springer-Verlag, Berlin, second edition, 2003.

24. M. Jeanblanc and M. Rutkowski. Default risk and hazard process. In Mathematica finance-Bachelier Congress, 2000 (Paris), Springer Finance, pages 281-312. Springer, Berlin, 2002

25. T. Jeulin. Grossissement d'une filtration et applications. In Séminaire de Probabilités, XIII (Univ. Strasbourg, Strasbourg, 1977/78), volume 721 of Lecture Notes in Math. pages 574-609. Springer, Berlin, 1979.

26. T. Jeulin. Comportement des semi-martingales dans un grossissement de filtration. $Z$. Wahrsch. Verw. Gebiete, 52(2):149-182, 1980.

27. J. A. Leon, R. Navarro, and D. Nualart. An anticipating calculus approach to the utility maximization of an insider. Mathematical Finance, 13(1):171-171, 2003.

28. D. Lépingle and J. Mémin. Sur l'intégrabilité uniforme des martingales exponentielles Zeitschrift für Wahrscheinlichkeitstheorie und Verwandte Gebiete, 42(3):175-203, 1978

29. E. Pardoux. Filtrage non linéaire et équations aux dérivées partielles stochastiques associées. In École d'Été de Probabilités de Saint-Flour XIX-1989, volume 1464 of Lecture Notes in Math., pages 67-163. Springer, Berlin, 1991.

30. H. Pham. On quadratic hedging in continuous time. Mathematical Methods for Operational Research, 51:315-339, 2000.

31. P. Protter. Stochastic integration and differential equations, A new approach, volume 21 of Appl. Math. Springer-Verlag, Berlin Heidelberg New York, $2^{\text {nd }}$ edition, 2001.

32. T. Rheinländer and M. Schweizer. On $L^{2}$-projections on a space of stochastic integrals. Ann. Probab., 25(4):1810-1831, 1997.

33. M. Schweizer. Option hedging for semimartingales. Stochastic Process. Appl., 37(2):339363, 1991. 